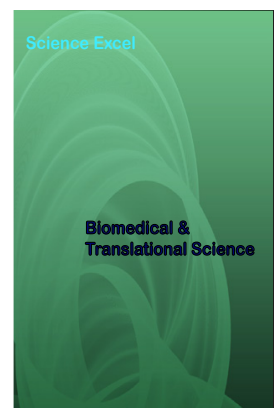

Correspondence

Isaac Rodriguez,

SweetBio, Inc., 460 S. Highland St. Floor 2,

Memphis, Tennessee, 38111 USA

Orcid.org/0000-0003-4632-5639

E-mail: isaac@sweetbio.com

Phone: 1-888-323-2015

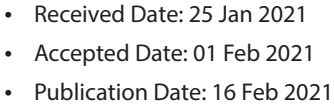

Keywords: bacteria, biomaterials, wound management, tissue engineering

\section{Copyright}

(C) 2021 Science Excel. This is an openaccess article distributed under the term of the Creative Commons Attribution 4.0 International license.

\title{
A Novel Bioengineered Wound Product with In Vitro Capabilities to Reduce Bacteria
}

\author{
Matthew Williams ${ }^{1}$, Isaac Rodriguez ${ }^{2 *}$, Axel Strombergsson'², Stefania Fabbri' \\ and Samantha Westgate ${ }^{1}$
}

'Perfectus Biomed Ltd., Techspace One, SciTech Daresbury, Keckwick Ln, Cheshire WA4 4AB, United Kingdom ${ }^{2}$ SweetBio, Inc., 460 S. Highland St. Floor 2, Memphis, Tennessee, 38111 USA

\begin{abstract}
Introduction: When microorganisms colonize a wound, there is a continuum which extends from contamination, to colonization and infection preventing a wound from healing. Biomaterials that reduce bacteria within the wound microenvironment can help progress it towards healing. Objective: This study aims to determine the in vitro bacteria reduction capabilities of a novel, commercially available bioengineered wound product (BWP) - a synthesis of gelatin (a highly purified collagen derivative), Manuka honey, and hydroxyapatite. Materials and methods: Using a direct contact method, the BWP and negative control were inoculated with suspensions of gram-positive (Staphylococcus aureus) and gram-negative (Pseudomonas aeruginosa) bacteria. After incubation for 30 minutes and 24 hours, recovery of viable organisms was performed. Results: There was a significant reduction $(99.99 \%)$ in bacterial load recovered from the BWP at 24 hours compared to the negative control. Additionally, the BWP caused a significant reduction in bacterial load at 24 hours compared to 30 minutes ( $97 \%$ and $64 \%$ reduction for Staphylococcus aureus and Pseudomonas aeruginosa, respectively). Conclusions: The significant reduction in bacterial load in vitro suggests that the product has the potential to help manage the pathogenic bioburden of a wound.
\end{abstract}

\section{Introduction}

When microorganisms colonize a wound, there is a continuum which extends from contamination, to colonization and infection preventing a wound from healing. Staphylococcus aureus, methicillinresistant Staphylococcus aureus (MRSA) and Pseudomonas aeruginosa are the predominant microbial strains that occur in patients with infected wounds [1]. Biomaterials that reduce bacteria within the wound microenvironment can help progress it towards healing. Compounds such as Manuka honey, silver, gold, and zinc have been integrated into wound dressings to achieve therapeutic effects on bacteria [2] Specifically, Manuka honey is an attractive and natural material that has been shown to inhibit bacterial growth $[3,4]$. In this study, an absorbable novel bioengineered wound product (BWP), a synthesis of gelatin (a highly purified collagen derivative), Manuka honey and hydroxyapatite, was evaluated for in vitro bacteria reduction capabilities against Staphylococcus aureus and Pseudomonas aeruginosa. While Manuka honey has been traditionally used for topical, short-term dressing applications, this novel BWP uniquely synthesizes Manuka honey into a longer-lasting, fully absorbable solid sheet for managing wounds.

\section{Materials and methods}

Preparation of the BWP and control product

The novel BWP (APIS ${ }^{\oplus}$, SweetBio, Inc., Memphis, TN) was prepared aseptically to $2 \mathrm{x}$ $2 \mathrm{~cm}$ sections. Each sample was immersed in sterile saline solution for one minute, turned over, and immersed in sterile saline for a further minute to ensure the BWP was fully hydrated. This preparation procedure was also replicated for the bacterial barrier negative control dressings $\left(\mathrm{N}-\mathrm{A}^{\oplus}\right.$ Knitted Viscose Dressing).

Bacteria reduction assessment via direct contact method

Suspensions of both gram-positive (Staphylococcus aureus ATCC ${ }^{\otimes} 6538^{\mathrm{rx}}$ ) and gram-negative (Pseudomonas aeruginosa ATCC $^{\otimes} 9027^{\mathrm{TN}}$ ) bacteria were prepared to $1 \mathrm{x}$ $10^{6} \pm 5 \times 10^{5} \mathrm{CFUmL}^{-1}$ in Tryptic Soy Broth (Scientific Laboratory Supplies, UK). The inoculum was enumerated by performing 10 fold dilutions and plating out the resulting suspensions onto Tryptic Soy Agar (TSA, Scientific Laboratory Supplies, UK). One hundred microliters of $1 \times 10^{6} \pm 5 \times 10^{5}$ $\mathrm{CFUmL}^{-1}$ bacterial suspension was used to inoculate the BWP and control samples. Samples were incubated for 30 minutes and 
24 hours at $37 \pm 2^{\circ} \mathrm{C}$. Following each incubation period, the BWP and control samples were placed into $3 \mathrm{~mL}$ of Quench neutralizing solution (Tween $8030 \mathrm{~g} / \mathrm{L}$, Lecithin $30 \mathrm{~g} / \mathrm{L}$, L-Histidine $1 \mathrm{~g} / \mathrm{L}$ and Sodium thiosulphate $4 \mathrm{~g} / \mathrm{L}$ in distilled water ( $\mathrm{pH} 7)$ ) and sonicated using a sonicating water bath (VWR, UK) for five minutes to recover any viable organisms. Microorganisms were enumerated by performing 10 -fold dilutions of the resultant suspensions and plating onto TSA in duplicate. All tests were performed in triplicate.

Statistical analysis

Average $\log 10 \mathrm{CFUmL}^{-1}$ bacterial recoveries and average $\log 10 \mathrm{CFUmL} \mathrm{H}^{-1}$ reductions, compared to the negative control, are presented as mean \pm standard deviation (SD) from three independent replicates, with duplicate repeats for each independent replicate. The minimum limit of detection for this study was 1 Log. A Student's unpaired t-test (twotailed) was used to assess statistical differences between the Log10CFUmL-1 recovery data from the negative control and BWP samples. Data was considered statistically significant when $\mathrm{p}<0.05$.

\section{Results}

\section{Staphylococcus aureus}

Following 24 hours incubation, the quantity of bacteria recovered from the negative control rose from $4.61 \pm 0.04$ $\log 10 \mathrm{CFUmL}^{-1}$ (30 minutes) to $6.85 \pm 0.19 \log 10 \mathrm{CFUmL}^{-1}(24$ hours) while the quantity of viable material recovered from the BWP reduced from $4.49 \pm 0.03 \log 10 \mathrm{CFUmL} \mathrm{Cm}^{-1}$ to $2.89 \pm$ $0.19 \log 10 \mathrm{CFUmL}^{-1}$ (Figure 1). This was an average reduction of $99.99 \%$ (3.96 $\pm 0.19 \log 10 \mathrm{CFUmL}^{-1}$ reduction) compared to the negative control at 24 hours $(p<0.001)$.

\section{Pseudomonas aeruginosa}

Following 24 hours incubation, the quantity of bacteria recovered from the negative control rose from $4.83 \pm 0.10$ $\log 10 \mathrm{CFUmL}^{-1}$ (30 minutes) to $8.42 \pm 0.05 \log 10 \mathrm{CFUmL}^{-1}(24$ hours) while the quantity of viable material recovered from the BWP reduced from $4.65 \pm 0.04 \log 10 \mathrm{CFUmL}^{-1}$ to $4.21 \pm 0.03$ $\log 10 \mathrm{CFUmL}^{-1}$ (Figure 1). This was an average reduction of 99.99\% (4.22 $\pm 0.03 \log 10 \mathrm{CFUmL}^{-1}$ reduction) compared to the negative control at 24 hours $(\mathrm{p}<0.001)$.

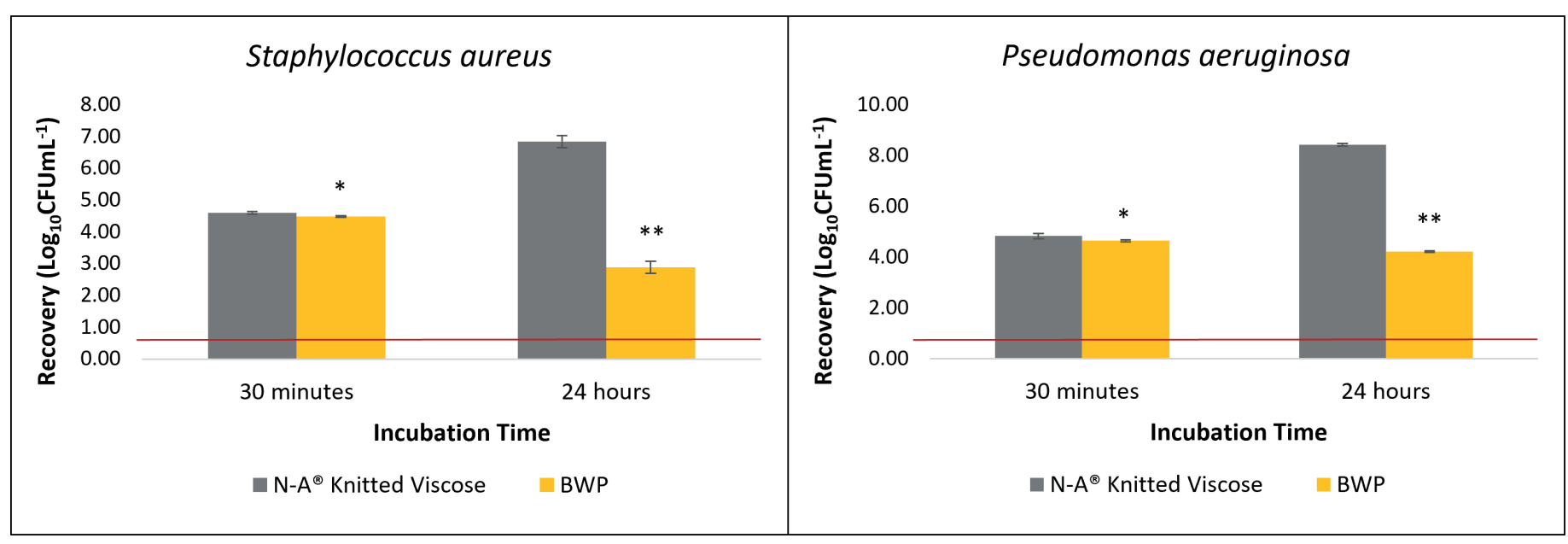

Figure 1. Quantity of viable Staphylococcus aureus (left) and Pseudomonas aeruginosa (right) recovered from the BWP and control items after 30 minutes and 24 hours incubation. The error bars indicate the standard deviation of the mean. The red line represents the limit of detection $(1$ $\log ) *=p<0.05, * *=p<0.001$.

Table 1. Average recovery of viable Staphylococcus aureus and Pseudomonas aeruginosa following 30 minutes and 24 hours treatment with the BWP, compared to the negative control. $\mathrm{CFU}=$ colony forming units, $\mathrm{SD}=$ standard deviation, $\mathrm{N} / \mathrm{A}=$ not applicable. $*=\mathrm{p}<0.05, * *=\mathrm{p}<$ 0.001 .

\begin{tabular}{|c|c|c|c|c|c|}
\hline \multirow[t]{2}{*}{ Organism } & \multirow[t]{2}{*}{ Test Item } & \multicolumn{2}{|c|}{$\begin{array}{l}\text { Average Log Recovery } \\
\left(\log 10 \mathrm{CFUmL}^{-1} \pm \mathrm{SD}\right)\end{array}$} & \multicolumn{2}{|c|}{$\begin{array}{l}\text { Average Log Reduction } \\
\left(\log 10 \mathrm{CFUmL}^{-1} \pm \mathrm{SD}\right)\end{array}$} \\
\hline & & 30 minutes & 24 hours & 30 minutes & 24 hours \\
\hline \multirow{2}{*}{$\begin{array}{l}\text { Staphylococcus } \\
\text { aureus }\end{array}$} & N-A ${ }^{\circledR}$ Knitted Viscose & $4.61 \pm 0.04$ & $6.85 \pm 0.19$ & N/A & N/A \\
\hline & BWP & $4.49 \pm 0.03$ & $2.89 \pm 0.19$ & $0.12 \pm 0.03 *$ & $3.96 \pm 0.19 * *$ \\
\hline \multirow{2}{*}{$\begin{array}{l}\text { Pseudomonas } \\
\text { aeruginosa }\end{array}$} & N-A ${ }^{\circledR}$ Knitted Viscose & $4.83 \pm 0.10$ & $8.42 \pm 0.05$ & $\mathrm{~N} / \mathrm{A}$ & N/A \\
\hline & BWP & $4.65 \pm 0.04$ & $4.21 \pm 0.03$ & $0.18 \pm 0.04^{*}$ & $4.22 \pm 0.03 * *$ \\
\hline
\end{tabular}




\section{Discussion and Conclusion}

Between 30 minutes and 24 hours, the bacterial load recovered from the $\mathrm{N}-\mathrm{A}^{\oplus}$ Knitted Viscose negative control dressing increased, demonstrating that bacterial growth was supported by this product. A significant reduction (99.99\%) in bacterial load recovered from the BWP at 24 hours compared to the negative control at 24 hours demonstrated, at minimum, bacteriostatic properties of the BWP. In addition, the BWP caused a significant reduction in bacterial load at 24 hours compared to 30 minutes $(97 \%$ and $64 \%$ reduction for Staphylococcus aureus and Pseudomonas aeruginosa, respectively), demonstrating a slight antibacterial impact of the BWP. The significant reduction in bacterial load in vitro suggests that the product has the potential to help manage the pathogenic bioburden of a wound. Future in vitro studies will assess if the BWP has the potential to be antimicrobial or serve as a microbial barrier.

\section{Acknowledgements and funding sources}

This study was funded by SweetBio, Inc. (Memphis, TN). Perfectus Biomed Ltd. was contracted to design and conduct the studies.

\section{Conflict of interest}

I.R. and A.S. are executives of SweetBio, Inc. I.R. is a coinventor of the BWP technology. All other authors have no conflict of interest to declare. The content of this article was expressly written by the authors listed. No ghostwriters were used to write the article.

\section{References}

1. Cardona AF, Wilson SE. Skin and soft-tissue infections: a critical review and the role of telavancin in their treatment. J Clin Infect Dis 2015;61 Suppl 2:S69-78.

2. Negut I, Grumezescu V, Grumezescu AM. Treatment strategies for infected wounds. Molecules 2018;23(9):2392.

3. Molan P, Rhodes T. Honey: A biologic wound dressing. Wounds 2015;27:141-151

4. Alvarez-Suarez JM, Gasparrini M, Forbes-Hernandez TY, Mazzoni L, Giampieri F. The composition and biological activity of honey: A focus on Manuka Honey. Foods 2014; 3:420-432. 